\title{
Prognostic value of miR-17-5 $p$ in gastrointestinal cancers: a systematic review and meta-analysis
}

This article was published in the following Dove Press journal: OncoTargets and Therapy

\author{
Zeyu Wang' \\ Jing Zhang ${ }^{2}$ \\ Zhiguang Zhang² \\ Yong Jiang ${ }^{2}$ \\ Man $\mathrm{Li}^{2}$ \\ Qian $\mathrm{Li}^{2}$ \\ Lu Bai \\ Dongying Yao' \\ Miao Wang \\ Xiaoping Wang' \\ 'Department of Gastroenterology, \\ Shanghai University of Medicine \& \\ Health Sciences Affiliated Zhoupu \\ Hospital, Shanghai, 201318, China; \\ ${ }^{2}$ Department of Gastroenterology, \\ The Second Hospital of Tianjin \\ Medical University, Tianjin, \\ 30021 I, China
}

\begin{abstract}
Background: There are accumulating studies investigating the aberrant expression of microRNAs in tumor patients. As an important member of miR-17/92 cluster, miR-17-5 $p$ has been identified as a potential prognostic factor for survival in tumor patients. We conducted this meta-analysis aimed to assess the effect of miR-17-5 $\mathrm{p}$ as a prognostic biomarker for gastrointestinal tumor patients.
\end{abstract}

Materials and methods: Eligible studies were enrolled by searching the online databases of PubMed, Embase, Web of Science, China National Knowledge Infrastructure, and WanFang Data until September 2017. We calculated pooled hazard ratios (HRs) and 95\% CI of miR-17-5 p for overall survival and disease-free survival.

Results: In the categorical variable analysis, we identified 11 studies with 1,279 patients. The pooled analyses suggested that overexpression of miR-17-5 $\mathrm{p}$ may predict poor overall survival $(\mathrm{HR}=1.86,95 \% \mathrm{CI}: 1.55-2.25, P<0.001)$ and disease-free survival $(\mathrm{HR}=1.43,95 \% \mathrm{CI}$ : $1.01-2.03, P=0.046)$ in patients with gastrointestinal tumors. Subgroup analysis showed the pooled HR of overall survival was more significant in tissue specimen, Asian patients, and digestive tract tumors. But there was no correlation between the outcomes and European patients.

Conclusions: This meta-analysis suggested that miR-17-5 $\mathrm{p}$ has predictive effects on overall survival and disease-free survival of patients with gastrointestinal tumors.

Keywords: miR-17-5 p, mircoRNA-17-5p, prognosis, survival, meta-analysis

\section{Introduction}

MicroRNAs (miRNAs) are a class of single-stranded noncoding RNAs 21-22 nucleotides in length processed from much longer primary transcripts. They may regulate up to $60 \%$ of human protein-coding genes. ${ }^{1}$ MiRNAs participate in crucial biological processes such as cell proliferation, differentiation, apoptosis, and tumorigenesis. ${ }^{2-4}$ MiR-17/92 cluster is one of the most popularly researched gene clusters and miR-17-5 $\mathrm{p}$ is an important member of the miR-17 family belonging to the miR-17/92 cluster. The human genome miR-17/92 cluster has two paralogues of the main cluster: the miR-106b/25 and the miR-106a/363 clusters. MiR-17/92 and miR-106b/25 are expressed abundantly in a wide spectrum of tissues but miR-106a/363 is expressed at lower levels. Together, these three miRNA clusters represent a combined total of 15 miRNAs that form four "seed" families: the miR-17 family, the miR-18 family, the miR-19 family, and the miR-92 family. ${ }^{5}$ The miR-17/92 cluster encodes six miRNAs including miR-17, miR-18a, miR19a, mir19b, miR-20a, and miR-92a. ${ }^{6}$ The recent studies showed that miRNAs are associated with prognosis in various cancers, suggesting that they could be used as prognostic classifiers to predict prognosis and guide therapeutic decisions. ${ }^{7-9}$ MiR-17-5 $\mathrm{p}$ was identified to have oncogenic or suppressive ability in several cancers. Many researchers have identified that the members
Correspondence: Xiaoping Wang Department of Gastroenterology, Shanghai University of Medicine \& Health Sciences Affiliated Zhoupu Hospital, Number 1500, Zhouyuan Road, Pudong District, Shanghai, 201318, China Tel +86 I592123 448I Emailxhnk00I@I63.com 
of miR-17/92 cluster are closely connected with diagnosis and prognosis of many kinds of tumors. ${ }^{10-13}$ As the important member of miR-17/92 cluster, the influence of miR-17-5 $p$ on the prognosis of glioblastoma, gastric carcinoma, hepatocellular carcinoma, lung cancer, pancreatic cancer, and malignant mesothelioma have been reported. ${ }^{14-22}$

However, there were still insignificant or opposite results. Therefore, we performed this meta-analysis to get a better understanding of the prognostic effect of miR-17-5 $p$ on gastrointestinal cancer patients.

\section{Materials and methods}

\section{Search strategy}

The systematic literature search was carried out through the online databases of PubMed, Embase, Web of science, China National Knowledge Infrastructure (CNKI), and Wan Fang to retrieve eligible studies till September 2017. The keywords of "miR-17-5 p", “miR17-5p", “microRNA17-5p", and "microRNA-17-5 p" were used to select the essays. Additionally, a manual search was conducted using all review articles on this topic. The researching of database was implemented by the authors QL and LB. The diagram of the study selection process is presented in Figure 1.

\section{Data extraction}

The investigators (QL and LB) performed data extraction using a standard data extraction form to determine eligibility for inclusion and extract data. Discrepancies were adjudged by the third investigator (ML) until consensus was achieved. The following inclusion criteria were used to select eligible studies in our meta-analysis: 1) patients with gastrointestinal cancers are included; 2) publication details: the name of tumors, first author's last name, and publication year; 3) characteristics of the studied population: follow-up time and stage of disease; 4) materials and methods of experiments; 5) miR-17-5 p assessment and cutoff definition; and 6) hazard ratios (HRs) of elevated miR-17-5 p for overall survival (OS) and disease-free survival (DFS) as well as their 95\% CI and $P$-values. The HRs and their 95\% CIs were performed in the original articles; if the HRs were not available, data were calculated following Tierney et al's method.

\section{Quality assessment}

We used Newcastle-Ottawa scale to assess the quality of enrolled studies. There are three aspects in Newcastle-Ottawa scale: the selection of the study groups, the comparability of the groups, and measuring of outcomes. The score of this scale is ranged from 0 to 9 .

\section{Statistical analysis}

To avoid calculating error, we pooled only HRs with their corresponding $95 \%$ CIs given explicitly in the publications in the analyses. Cochran's Q test and Higgin's $I^{2}$ statistic were

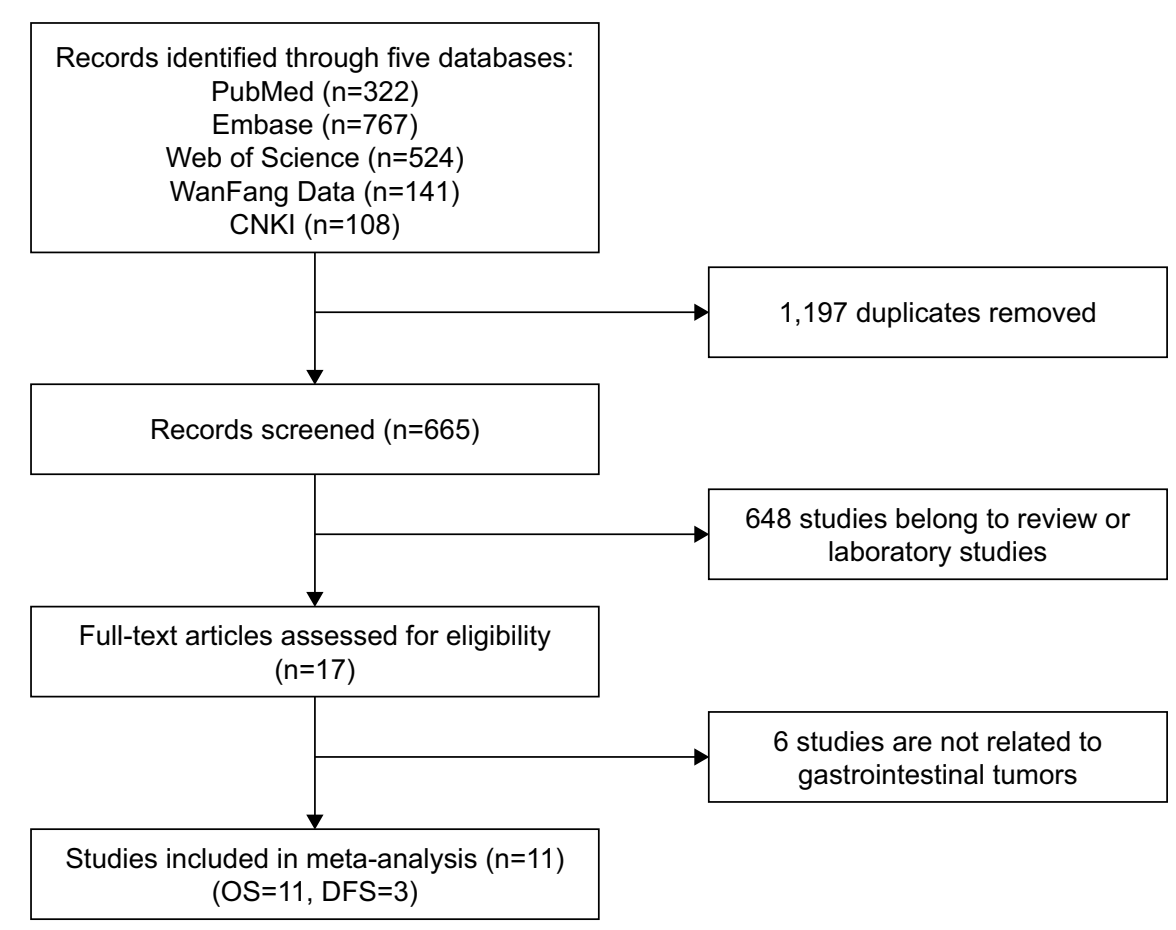

Figure I Flow diagram of the study selection process.

Abbreviations: OS, overall survival; DFS, disease-free survival. 
used to measure the heterogeneity between studies. If $P>0.1$ or $I^{2}<50 \%$, fixed-effects model (Mantel-Haenszel test) was used; otherwise, random-effects model (DerSimonian and Laird method) was chosen. Sensitivity analysis was conducted by omitting one single research at a time and recounting HRs and 95\% CIs to assess the stability of the meta-analysis results. Publication bias among the included studies was evaluated by Egger's and Begg's statistical tests. All $P$-values were two-sided. All statistical tests were analyzed by STATA 11.0 (STATA Corporation, College Station, TX, USA).

\section{Results}

\section{Description of included studies}

The flowchart of detailed searching process is illustrated in Figure 1. We used the searching strategy described above to search the online databases of PubMed, Embase, Web of Science, CNKI, and Wan Fang. A total of 1,862 records were initially collected by our literature search strategy. After manually screening the titles and abstracts, our investigators excluded duplicates and articles that failed to meet the aim of our study. Then 17 records were assessed for full-text review. Finally, eleven ${ }^{15-19,23-28}$ studies that evaluated the prognostic effect of miR-17-5 p for OS and three ${ }^{16,25,26}$ studies for DFS were included.

\section{Characteristics of eligible studies}

A total of 1,279 cases from China, Japan, Spain, and Hungary were included to evaluate the relationship between miR-17-5 p expression and tumor prognosis. The patients were diagnosed with gastrointestinal cancers, including colorectal carcinoma, hepatocellular carcinoma, pancreatic carcinoma, gastric carcinoma, esophageal squamous cell carcinoma, and cholangiocarcinoma. Tissue and blood samples were used to determine expression levels of miR-17-5 $p$ while quantitative real-time polymerase chain reaction had been used in all studies. The most frequently applied cutoff value was the median which was used in seven studies and others were different. Other basic characteristics are all listed in Table 1. All the HRs and their 95\% CIs in the collected articles are shown in Table 2.

\section{Qualitative assessment}

After using Newcastle-Ottawa scale to assess the quality of studies, the study quality varied from 5 to 9 ; the studies that achieved a score $>6$ were included. The clinicopathological characteristics of the eligible studies are summarized in Table 3.

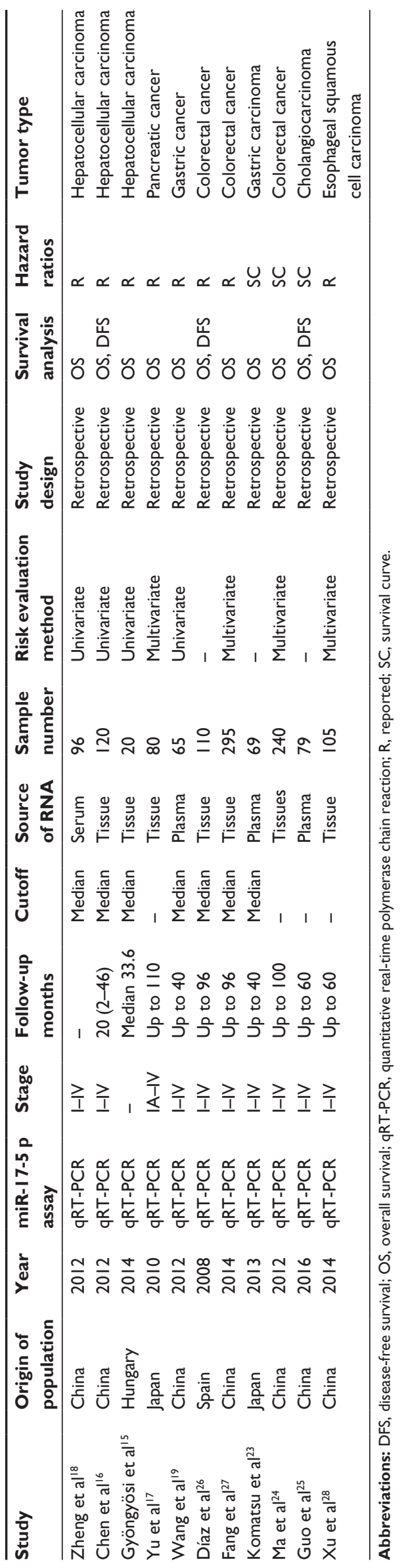


Table 2 Summary of HRs and their $95 \% \mathrm{Cl}$

\begin{tabular}{|c|c|c|c|c|c|c|c|c|c|}
\hline Study & $\begin{array}{l}\text { Origin of } \\
\text { population }\end{array}$ & Year & Disease & HR & OS $95 \% \mathrm{Cl}$ & $P$-value & HR & $\begin{array}{l}\text { DFS } \\
95 \% \mathrm{Cl}\end{array}$ & $P$-value \\
\hline Komatsu et $\mathrm{al}^{23}$ & Japan & 2013 & Gastric carcinoma & 1.33 & $0.15-11.79$ & 0.7978 & NR & NR & NR \\
\hline Ma et $\mathrm{al}^{24}$ & China & 2012 & Colorectal cancer & 2.24 & $1.58-3.18$ & $<0.001$ & NR & NR & NR \\
\hline Guo et $\mathrm{al}^{25}$ & China & 2016 & Cholangiocarcinoma & 1.55 & $0.83-2.89$ & 0.169 & 1.09 & $0.57-2.06$ & 0.7 \\
\hline Zheng et $\mathrm{al}^{18}$ & China & 2012 & Hepatocellular carcinoma & 2.192 & $1.024-4.69 \mid$ & 0.043 & NR & NR & NR \\
\hline Chen et $\mathrm{al}^{16}$ & China & 2012 & Hepatocellular carcinoma & 4.96 & $1.78-13.82$ & 0.002 & 1.79 & $1.14-2.98$ & 0.0 \\
\hline Gyöngyösi et al ${ }^{15}$ & Hungary & 2014 & Hepatocellular carcinoma & 1.09 & $0.37-3.26$ & 0.88 & NR & NR & NR \\
\hline Yu et al ${ }^{17}$ & Japan & 2016 & Pancreatic cancer & 0.9 & $0.4-1.7$ & 0.13 & NR & NR & NR \\
\hline Wang et al ${ }^{19}$ & China & 2012 & Gastric cancer & 1.785 & I.I I0-2.870 & 0.017 & NR & NR & NR \\
\hline Díaz et $\mathrm{al}^{26}$ & Spain & 2008 & Colorectal cancer & 1.06 & $0.47-2.39$ & 0.9 & 1.13 & $0.48-2.68$ & 0.7 \\
\hline Fang et $\mathrm{al}^{27}$ & China & 2014 & Colorectal cancer & 1.9 & I.195-3.022 & 0.007 & NR & NR & NR \\
\hline Xu et $\left.a\right|^{28}$ & China & 2014 & $\begin{array}{l}\text { Esophageal squamous } \\
\text { cell carcinoma }\end{array}$ & 2.849 & $1.258-6.455$ & 0.012 & NR & NR & NR \\
\hline
\end{tabular}

Abbreviations: DFS, disease-free survival; HR, hazard ratio; NR, not reported; OS, overall survival.

\section{Meta-analysis results}

All the studies were pooled into this meta-analysis, and the result is shown in Figure 2. As no heterogeneity was found in OS research $\left(I^{2}=22.6 \%, P=0.228\right)$ and DFS research $\left(I^{2}=0 \%, P=0.40\right)$, therefore, fixed model was applied. The result indicated that overexpression of miR-17-5 p significantly predicted poor OS $(\mathrm{HR}=1.86,95 \% \mathrm{CI}$ : $1.55-2.25$; Figure 2A), and the effect reached the level of statistical significance $(P<0.001)$. DFS was reported in three studies. The pooled HR of DFS was 1.43 (95\% CI: 1.01-2.03, $P=0.046$; Figure 2), indicating that the high level of miR-17-5 p expression was related to poor DFS. Detailed analyses were also carried out. We further researched the meta-analysis of miR-17-5 $\mathrm{p}$ expression and OS in different cancer types: three studies including colorectal carcinoma, three studies including hepatocellular carcinoma, two studies including gastric carcinoma, one study including pancreatic carcinoma,

Table 3 Quality assessment based on the Newcastle-Ottawa scale

\begin{tabular}{|c|c|c|c|c|}
\hline & Selection & Comparability & Outcome & $\begin{array}{l}\text { Total } \\
\text { score }\end{array}$ \\
\hline Komatsu et al/201 $3^{23}$ & 3 & I & 3 & 7 \\
\hline Zheng et $\mathrm{al} / 2013^{18}$ & 3 & 2 & I & 7 \\
\hline Chen et al/2012 $2^{16}$ & 4 & 2 & 3 & 9 \\
\hline Gyöngyösi et al/201415 & 4 & I & 2 & 7 \\
\hline Yu et al/2016 $6^{17}$ & 4 & I & 2 & 7 \\
\hline Ma et al/201 $2^{24}$ & 4 & 2 & 3 & 9 \\
\hline Guo et al/2016 $6^{25}$ & 3 & I & 2 & 6 \\
\hline Díaz et al/2008 26 & 4 & 2 & I & 7 \\
\hline Xu et al/201 $4^{28}$ & 4 & 1 & 2 & 7 \\
\hline Fang et al/201 $4^{27}$ & 4 & 2 & 2 & 8 \\
\hline Wang et al/201 $2^{19}$ & 4 & 2 & 2 & 8 \\
\hline
\end{tabular}

Notes: Selection: representativeness of studies (maximum score of 4). Comparability: comparability of studies based on the design or analysis (maximum score of 2). Outcome: assessment of outcome and follow-up (maximum score of 3 ). one study including esophageal squamous cell carcinoma, and one study including cholangiocarcinoma (Figure 2). No obvious heterogeneity was found in each subgroup. The results prompt that overexpression of miR-17-5 $\mathrm{p}$ was related to poor OS in colorectal carcinoma, hepatocellular carcinoma, and gastric carcinoma. But the difference was not significant in pancreatic carcinoma.

Subgroup analyses were conducted according to the main characteristics. The results showed that the predictive role of miR-17-5p was significant in blood specimen $(\mathrm{HR}=1.70$, 95\% CI: 1.23-2.34, fixed-effects model), tissue specimen (HR $=1.90,95 \% \mathrm{CI}: 1.29-2.80$, random-effects model $)$, Asian patients (HR $=1.98,95 \%$ CI: 1.61-2.38, fixed-effects model), digestive tract tumors (including gastric carcinoma, colorectal carcinoma, esophageal squamous cell carcinoma; $\mathrm{HR}=1.97,95 \%$ CI: 1.58-2.45, fixed-effects model), hepatobiliary system, and pancreas tumor (including hepatocellular carcinoma, pancreatic carcinoma, and cholangiocarcinoma; HR $=1.62$, 95\% CI: 1.14-2.31, fixed-effects model). The association between overexpressed miR-17-5 $\mathrm{p}$ and poor OS was not significant in the subgroup of European patients (Table 4).

\section{Sensitivity analysis}

Considering the number of the OS studies was more than ten, a sensitivity analysis was performed to assess the stability of the meta-analysis results. After omitting one study each time, the pooled HRs were calculated again. The stability of the entire study was not influenced by one individual study (Figure 3).

\section{Publication bias assessment}

The Begg's funnel plot and Egger's test were applied to detect publication bias in this OS and DFS meta-analysis among 
included studies (Figure 4). In OS and DFS meta-analysis, the values of Begg's regression were 0.558 and 0.373 . The result suggests that no obvious risk of publication bias was found in the meta-analysis.

\section{Discussion}

The function of miR-17-92 cluster had been reported in many different aspects. As an important member of miR-17-92 cluster, miR-17-5 $\mathrm{p}$ had been demonstrated playing an important role in aging of brain, heart, and bone. According to the recent researches, the review of Dellago summed up that miR-17-5 $\mathrm{p}$ is at the crossroads of aging, longevity, and cancer and can be used a biomarker or a therapeutic tool. ${ }^{29}$

Emerging evidence indicate that the miR-17-92 cluster may play an important role in tumorigenesis as a novel class of oncogenes. ${ }^{30,31}$ MiR-17-5 $\mathrm{p}$ is one of the main effectors of the miR-17-92 cluster components. MiR-17-5 p, as a novel biomarker of prognosis in many types of cancer, has generated much interest. Many studies suggest that overexpression of miR-17-5 p plays important roles in increasing cell proliferation, migration, and invasion. MiR-17-5 p functions as a tumor suppressor in biological activities. Cheng et al revealed that miR-17-5 p promotes tumor cell migration by upregulating a calcium-activated potassium channel subunit alpha 1 (KCa1.1) in malignant pleural mesothelioma. ${ }^{32} \mathrm{Li}$ et al discovered that overexpression of miR-17-5 p in MCF-7 cells promote the invasive and migratory abilities by targeting HBP1 through $\beta$-catenin pathway. ${ }^{33}$

In addition, the role of miR-17-5 $\mathrm{p}$ as an oncogene or tumor suppressor has also been reported in cervical cancer ${ }^{34}$

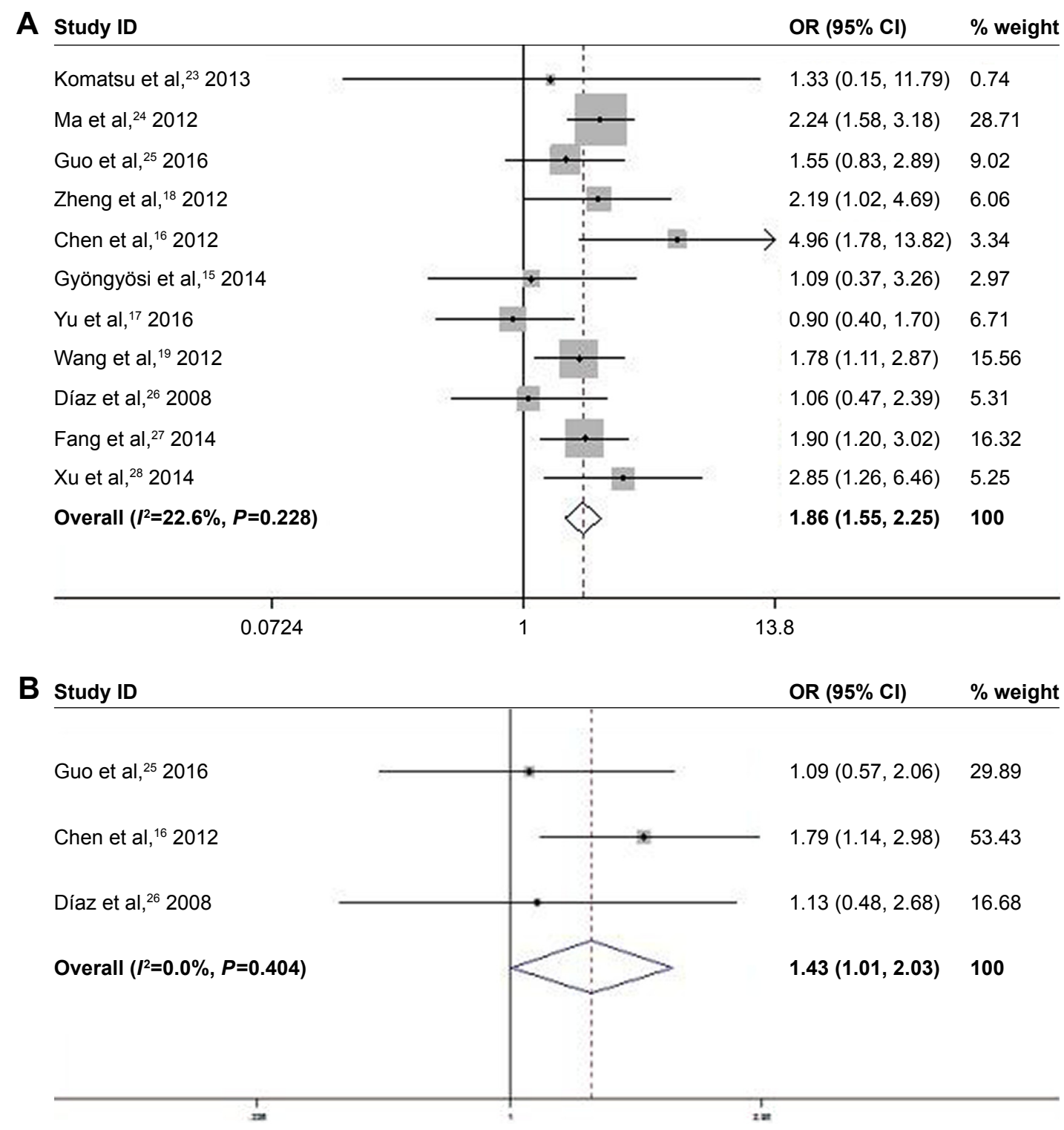

Figure 2 (Continued) 


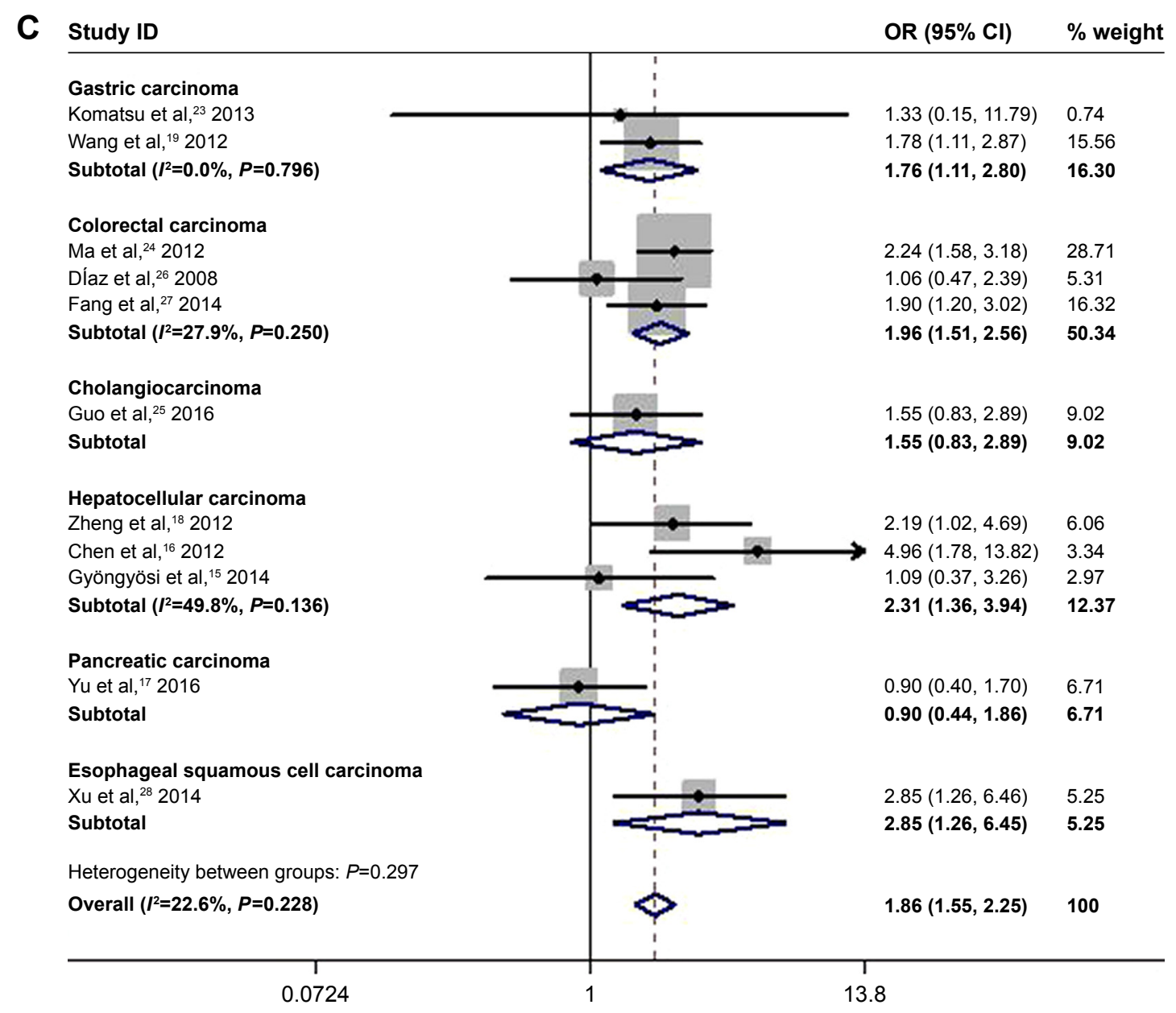

Figure 2 Forest plot of overall survival analysis and disease-free survival analysis.

Notes: (A) Meta-analysis of miR-17-5 p expression and overall survival. (B) Meta-analysis of miR-17-5 p expression and disease-free survival. (C) Meta-analysis of miR-17-5 P expression and overall survival in different kinds of tumors.

and breast cancer. ${ }^{35,36}$ Furthermore, in gastrointestinal tumors, miR-17-5 p-induced phosphorylation of HSP27 enhances the migration of HCCs through the p38 MAPK pathway. ${ }^{37}$ In gastric cancer, miR-17-5 $\mathrm{p}$ downregulated TGFBR2 expression, thereby promoting cell growth and migration. ${ }^{38}$
GFRalpha2 influences cell growth and apoptosis through regulating tumor gene PTEN via mir-17-5 $p$ in pancreatic cancer. ${ }^{39}$

Up to now, many studies have reported that miR-17-5 p influences the effectiveness of the chemotherapy in some

Table 4 Meta-analysis of overall survival and subgroup analysis

\begin{tabular}{|c|c|c|c|c|c|c|}
\hline \multirow[t]{2}{*}{ Stratified study } & \multirow{2}{*}{$\begin{array}{l}\text { No of } \\
\text { studies }\end{array}$} & \multirow[t]{2}{*}{ HR (95\% Cl) } & \multirow[t]{2}{*}{ Model } & \multirow[t]{2}{*}{$P$-value } & \multicolumn{2}{|c|}{ Heterogeneity } \\
\hline & & & & & $P^{2}(\%)$ & $\overline{P \text {-value }}$ \\
\hline \multicolumn{7}{|l|}{ Year } \\
\hline$>2012$ & 6 & $2.05(\mathrm{I} .6 \mathrm{I}-2.6 \mathrm{I})$ & Fixed & $<0.001$ & 18.5 & 0.293 \\
\hline$\leq 2012$ & 5 & $1.62(1.21-2.18)$ & Fixed & 0.001 & 25.2 & 0.253 \\
\hline \multicolumn{7}{|l|}{ Race } \\
\hline Asian & 9 & $1.98(1.61-2.38)$ & Fixed & $<0.001$ & 19 & 0.274 \\
\hline European & 2 & $1.07(0.56-2.05)$ & Fixed & 0.837 & 0 & 0.968 \\
\hline \multicolumn{7}{|l|}{ Material } \\
\hline Blood & 5 & $1.70(1.23-2.34)$ & Fixed & 0.001 & 0 & $0.87 I$ \\
\hline Tissue & 6 & $1.90(1.29-2.80)$ & Random & $<0.001$ & 55.3 & 0.048 \\
\hline \multicolumn{7}{|l|}{ Cancer type } \\
\hline Digestive tract tumors & 6 & $1.97(1.58-2.45)$ & Fixed & $<0.001$ & 0 & 0.572 \\
\hline $\begin{array}{l}\text { Hepatobiliary system } \\
\text { and pancreas tumors }\end{array}$ & 5 & $1.62(1.14-2.31)$ & Fixed & 0.007 & 51.5 & 0.083 \\
\hline
\end{tabular}

Abbreviation: $\mathrm{HR}$, hazard ratio. 


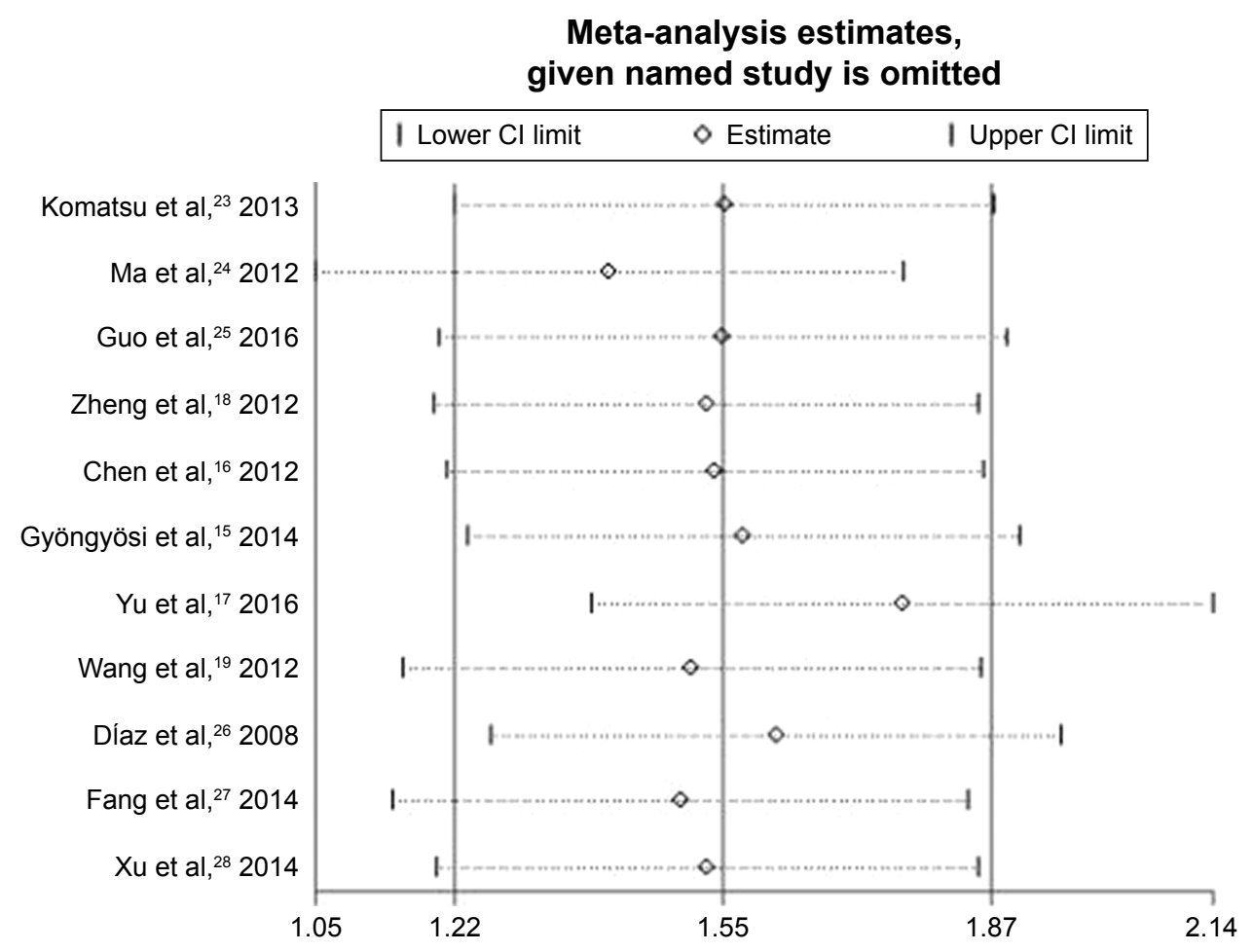

Figure 3 Result of sensitivity analyses by omitting one study in each turn.

tumors in vivo. In Yan's research, inhibiting miR-17-5 p expression increased chemosensitivity to gemcitabine in the Panc- 1 and BxPC3 cells. ${ }^{40}$ Fang et al found that miRNA17-5 p promotes tumor metastasis and chemotherapeutic drug resistance of colorectal cancer by downregulating the expression of PTEN. ${ }^{27}$ Chatterjee et al examined that miR-17-5 p downregulation contributes to paclitaxel resistance through altering Beclin1 expression in lung cancer. ${ }^{41}$ By performing a similar study in radiotherapy, Wu et al identified that miRNA-17-5 $\mathrm{p}$ regulated radiosensitivity in oral squamous cell carcinoma. ${ }^{42}$ The downregulated mir-17-5 $\mathrm{p}$ in esophageal adenocarcinoma cancer stem-like cells may promote the radioresistant phenotype. ${ }^{43}$

Recently, many studies reported that miR-17-5 $\mathrm{p}$ is associated with poor OS in many types of tumor. However, the sample size of these studies is relatively small. In most researches, ${ }^{14,16,18,19}$ miR-17-5 $\mathrm{p}$ indicates a poor prognosis in hepatocellular, lung, and gastric carcinomas. But there is still argument between the function of overexpression of miR-17-5 $\mathrm{p}$ and predicting the survival. In Yu et al's multivariate survival analyses of pancreatic cancer and Gyöngyösi et al's research of hepatocellular carcinoma, there was no
A

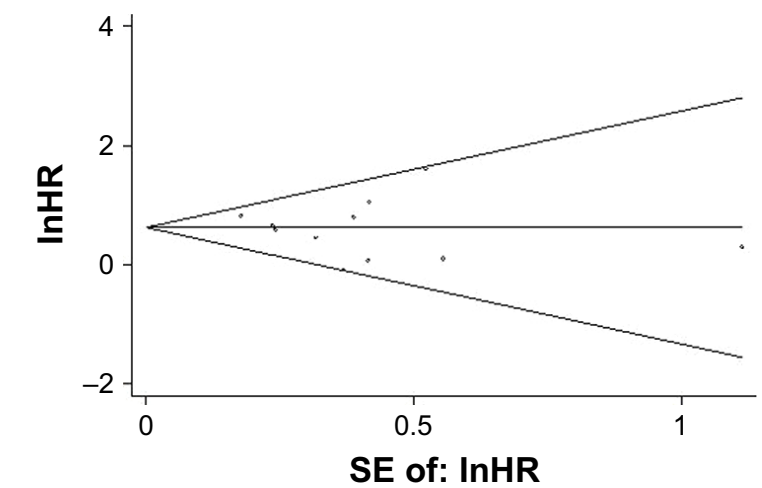

B Begg's funnel plot with
pseudo $95 \%$ confidence limits

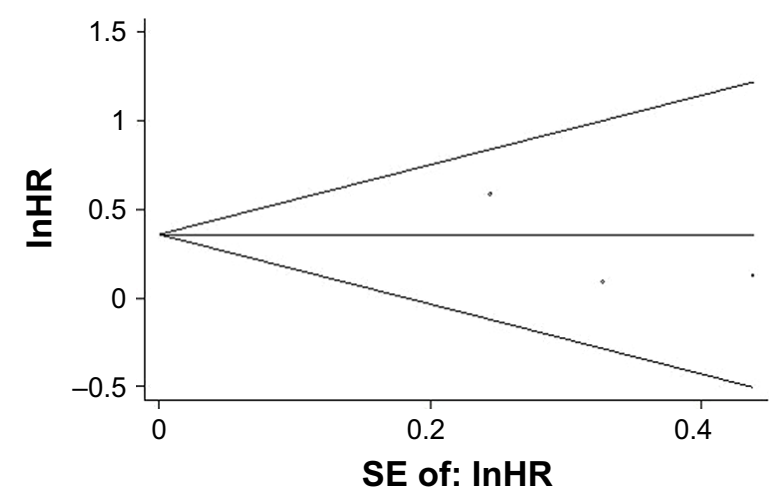

Figure 4 (A) Funnel plot of miR-17-5 p and overall survival. (B) Funnel plot of miR-17-5 $p$ and disease-free survival. Abbreviations: HR, hazard ratio; SE, standard error. 
significant association between overexpression of miR-17-5 $\mathrm{p}$ level and the OS time. ${ }^{15,17}$ In contrast, in Srinivasan et al's study of glioblastoma, ${ }^{20}$ overexpression of miR-17-5 $\mathrm{p}$ was proved to contribute to increase the patients' survival time. With the increasing number of researches related to miR-17-5 $\mathrm{p}$ in gastrointestinal tumors, we noted that half of researches about the prognostic value of miR-17-5 $p$ in gastrointestinal tumors were insignificant. So we conducted this meta-analysis aiming to better understand the association between miR-17-5 $\mathrm{p}$ expression and survival in gastrointestinal tumor patients. Our study included eleven researches and higher expression of miR-17-5 p predicted poor OS $(\mathrm{HR}=1.86,95 \% \mathrm{CI}$ : 1.55-2.25; fixed-effects model) and poor DFS (HR $=1.43$, 95\% CI: 1.01-2.03; fixed-effects model). Nevertheless, the present study has some limitations that require consideration. First, our study analyzed only unvaried factors; we did not stratify and analyze factors such as gender, treatment, and environmental variables due to lack of data. Second, the types of tumor are still deficient. Third, the analysis of the expression of miR-17-5 p and DFS is based only on three studies, one from each of three different cancer types including one study looking at plasma expression. Fourth, although our research shows that miR-17-5 $p$ is significantly correlated with the prognosis of gastrointestinal tumors, the clinical characteristics of different tumors may affect the combined results. The influence of different sample sources (tissue/serum) on the combined results should also be taken into consideration. Therefore, a more precise analysis needs to be performed.

\section{Conclusion}

In conclusion, the results of our meta-analysis support a potential prognostic role for miR-17-5 $\mathrm{p}$ in some cancers. Further studies should be performed to analyze the association between prognosis and other clinic characteristics.

\section{Disclosure}

The authors report no conflicts of interest in this work.

\section{References}

1. Fang Z, Yin S, Sun R, et al. miR-140-5p suppresses the proliferation, migration and invasion of gastric cancer by regulating YES1. Mol Cancer. 2017;16(1):139.

2. Yang W, Wei J, Guo T, Shen Y, Liu F. Knockdown of miR-210 decreases hypoxic glioma stem cells stemness and radioresistance. Exp Cell Res. 2014;326(1):22-35.

3. Shen Y, Gong JM, Zhou LL, Sheng JH. MiR-451 as a new tumor marker for gastric cancer. Oncotarget. 2017;8(34):56542-56545.

4. Shen J, Zhang J, Xiao M, Yang J, Zhang N. MiR-203 Suppresses Bladder Cancer Cell Growth and Targets the Twist1. Oncol Res. Epub 2017 Sep 6.
5. Mogilyansky E, Rigoutsos I. The miR-17/92 cluster: a comprehensive update on its genomics, genetics, functions and increasingly important and numerous roles in health and disease. Cell Death Differ. 2013; 20(12):1603-1614.

6. Feuermann Y, Robinson GW, Zhu BM, et al. The miR-17/92 cluster is targeted by STAT5 but dispensable for mammary development. Genesis. 2012;50(9):665-671.

7. Qin C, Huang RY, Wang ZX. Potential role of miR-100 in cancer diagnosis, prognosis, and therapy. Tumour Biol. 2015;36(3):1403-1409.

8. Wu MY, Fu J, Xiao X, Wu J, Wu RC. MiR-34a regulates therapy resistance by targeting $\mathrm{HDAC} 1$ and $\mathrm{HDAC} 7$ in breast cancer. Cancer Lett. 2014;354(2):311-319.

9. Chen AH, Qin YE, Tang WF, Tao J, Song HM, Zuo M. MiR-34a and miR-206 act as novel prognostic and therapy biomarkers in cervical cancer. Cancer Cell Int. 2017;17:63.

10. Su ZX, Zhao J, Rong ZH, Wu YG, Geng WM, Qin CK. Diagnostic and prognostic value of circulating miR-18a in the plasma of patients with gastric cancer. Tumour Biol. 2014;35(12):12119-12125.

11. Komatsu S, Ichikawa D, Takeshita H, et al. Circulating miR-18a: a sensitive cancer screening biomarker in human cancer. In Vivo. 2014;28(3): 293-297.

12. Ma Q, Peng Z, Wang L, et al. miR-19a correlates with poor prognosis of clear cell renal cell carcinoma patients via promoting cell proliferation and suppressing PTEN/SMAD4 expression. Int J Oncol. 2016; 49(6):2589-2599.

13. Yang $\mathrm{R}, \mathrm{Fu} \mathrm{Y}$, Zeng $\mathrm{Y}$, et al. Serum miR-20a is a promising biomarker for gastric cancer. Biomed Rep. 2017;6(4):429-434.

14. Chen Q, Si Q, Xiao S, et al. Prognostic significance of serum miR-17-5p in lung cancer. Med Oncol. 2013;30(1):353.

15. Gyöngyösi B, Végh É, Járay B, et al. Pretreatment MicroRNA Level and Outcome in Sorafenib-treated Hepatocellular Carcinoma. J Histochem Cytochem. 2014;62(8):547-555.

16. Chen L, Jiang M, Yuan W, Tang H. miR-17-5p as a novel prognostic marker for hepatocellular carcinoma. J Invest Surg. 2012;25(3): $156-161$.

17. Yu J, Ohuchida K, Mizumoto K, Fujita H, Nakata K, Tanaka M. MicroRNA miR-17-5p is overexpressed in pancreatic cancer, associated with a poor prognosis, and involved in cancer cell proliferation and invasion. Cancer Biol Ther. 2010;10(8):748-757.

18. Zheng J, Dong P, Gao S, Wang N, Yu F. High expression of serum miR-17-5p associated with poor prognosis in patients with hepatocellular carcinoma. Hepatogastroenterology. 2013;60(123):549-552.

19. Wang M, Gu H, Wang S, et al. Circulating miR-17-5p and miR-20a: molecular markers for gastric cancer. Mol Med Rep. 2012;5(6): 1514-1520.

20. Srinivasan S, Patric IR, Somasundaram K. A ten-microRNA expression signature predicts survival in glioblastoma. PLoS One. 2011; 6(3): 17438.

21. Busacca S, Germano S, de Cecco L, et al. MicroRNA signature of malignant mesothelioma with potential diagnostic and prognostic implications. Am J Respir Cell Mol Biol. 2010;42(3):312-319.

22. Zakrzewska M, Fendler W, Zakrzewski K, et al. Altered MicroRNA Expression Is Associated with Tumor Grade, Molecular Background and Outcome in Childhood Infratentorial Ependymoma. PLoS One. 2016;11(7):e158464.

23. Komatsu S, Ichikawa D, Tsujiura M, et al. Prognostic impact of circulating miR-21 in the plasma of patients with gastric carcinoma. Anticancer Res. 2013;33(1):271-276.

24. Ma Y, Zhang P, Wang F, et al. Elevated oncofoetal miR-17-5p expression regulates colorectal cancer progression by repressing its target gene P130. Nat Commun. 2012;3:1291.

25. Guo M, Tan M, Ye T. Expression of serum microRNA-17-5p in patients with cholangiocarcinoma and its clinical significance. Chinese Journal of Modern Medicine. 2016;26(19):42-46.

26. Díaz R, Silva J, García JM, et al. Deregulated expression of miR-106a predicts survival in human colon cancer patients. Genes Chromosomes Cancer. 2008;47(9):794-802. 
27. Fang L, Li H, Wang L, et al. MicroRNA-17-5p promotes chemotherapeutic drug resistance and tumour metastasis of colorectal cancer by repressing PTEN expression. Oncotarget. 2014;5(10):2974-2987.

28. Xu XL, Jiang YH, Feng JG, Su D, Chen PC, Mao WM. MicroRNA-17, microRNA-18a, and microRNA-19a are prognostic indicators in esophageal squamous cell carcinoma. Ann Thorac Surg. 2014;97(3): 1037-1045.

29. Dellago H, Bobbili MR, Grillari J. MicroRNA-17-5p: At the Crossroads of Cancer and Aging - A Mini-Review. Gerontology. 2017;63(1):20-28.

30. Liu D, Dong L, Liu Y, et al. A c-Myc/miR-17-5p feedback loop regulates metastasis and invasion of hepatocellular carcinoma. Tumour Biol. 2016;37(4):5039-5047.

31. Wang M, Gu H, Qian H, et al. miR-17-5p/20a are important markers for gastric cancer and murine double minute 2 participates in their functional regulation. Eur J Cancer. 2013;49(8):2010-2021.

32. Cheng YY, Wright CM, Kirschner MB, et al. KCa1.1, a calciumactivated potassium channel subunit alpha 1 , is targeted by miR-17-5p and modulates cell migration in malignant pleural mesothelioma. $\mathrm{Mol}$ Cancer. 2016;15(1):44.

33. Li H, Bian C, Liao L, Li J, Zhao RC. miR-17-5p promotes human breast cancer cell migration and invasion through suppression of HBP1. Breast Cancer Res Treat. 2011;126(3):565-575.

34. Wei Q, Li YX, Liu M, Li X, Tang H. MiR-17-5p targets TP53INP1 and regulates cell proliferation and apoptosis of cervical cancer cells. IUBMB Life. 2012;64(8):697-704.

35. Fan M, Sethuraman A, Brown M, Sun W, Pfeffer LM. Systematic analysis of metastasis-associated genes identifies miR-17-5p as a metastatic suppressor of basal-like breast cancer. Breast Cancer Res Treat. 2014;146(3):487-502.
36. Hossain A, Kuo MT, Saunders GF. Mir-17-5p regulates breast cancer cell proliferation by inhibiting translation of AIB1 mRNA. Mol Cell Biol. 2006;26(21):8191-8201.

37. Yang F, Yin Y, Wang F, et al. miR-17-5p Promotes migration of human hepatocellular carcinoma cells through the p38 mitogen-activated protein kinase-heat shock protein 27 pathway. Hepatology. 2010;51(5): 1614-1623.

38. Qu Y, Zhang H, Duan J, et al. MiR-17-5p regulates cell proliferation and migration by targeting transforming growth factor-beta receptor 2 in gastric cancer. Oncotarget. 2016;7(22):33286-33296.

39. Gu J, Wang D, Zhang J, et al. GFRalpha2 prompts cell growth and chemoresistance through down-regulating tumor suppressor gene PTEN via Mir-17-5p in pancreatic cancer. Cancer Lett. 2016;380(2): 434-441.

40. Yan HJ, Liu WS, Sun WH, et al. miR-17-5p inhibitor enhances chemosensitivity to gemcitabine via upregulating Bim expression in pancreatic cancer cells. Dig Dis Sci. 2012;57(12):3160-3167.

41. Chatterjee A, Chattopadhyay D, Chakrabarti G. miR-17-5p downregulation contributes to paclitaxel resistance of lung cancer cells through altering beclin1 expression. PLoS One. 2014;9(4):e95716.

42. Wu SY, Wu AT, Liu SH, Sy W, At W. MicroRNA-17-5p regulated apoptosis-related protein expression and radiosensitivity in oral squamous cell carcinoma caused by betel nut chewing. Oncotarget. 2016; 7(32):51482-51493.

43. Lynam-Lennon N, Heavey S, Sommerville G, et al. MicroRNA-17 is downregulated in esophageal adenocarcinoma cancer stem-like cells and promotes a radioresistant phenotype. Oncotarget. 2017;8(7): $11400-11413$
OncoTargets and Therapy

\section{Publish your work in this journal}

OncoTargets and Therapy is an international, peer-reviewed, open access journal focusing on the pathological basis of all cancers, potential targets for therapy and treatment protocols employed to improve the management of cancer patients. The journal also focuses on the impact of management programs and new therapeutic agents and protocols on

\section{Dovepress}

patient perspectives such as quality of life, adherence and satisfaction. The manuscript management system is completely online and includes a very quick and fair peer-review system, which is all easy to use. Visit http://www.dovepress.com/testimonials.php to read real quotes from published authors. 